\title{
APPROACHING THE CHIRAL POINT IN TWO-FLAVOUR LATTICE SIMULATIONS*
}

\author{
Stefano LotTini \\ for the ALPHA Collaboration \\ NIC, DESY, Platanenallee 6, 15738 Zeuthen, Germany \\ (Received June 17, 2014)
}

\begin{abstract}
We investigate the behaviour of the pion decay constant and the pion mass in two-flavour lattice QCD, with the physical and chiral points as ultimate goal. Measurements come from the ensembles generated by the CLS initiative using the $\mathcal{O}(a)$-improved Wilson formulation, with lattice spacing down to about 0.05 fermi and pion masses as low as $190 \mathrm{MeV}$. The applicability of $\mathrm{SU}(2)$ chiral perturbation theory is investigated, and various functional forms, and their range of validity, are compared. The physical scale is set through the kaon decay constant, whose measurement is enabled by inserting a third, heavier valence strange quark.
\end{abstract}

DOI:10.5506/APhysPolBSupp.7.565

PACS numbers: 12.38.Gc, 12.39.Fe, 12.38.- -t

\section{Introduction}

The only well-established, first-principle tool to explore the low-energy dynamics of QCD without additional assumptions is, to date, Lattice QCD; it is the formulation of the theory on a discrete spacetime that acts as a regulator while opening the way to numerical computations, thus enabling the study of intrinsically nonperturbative features of the theory.

On the other hand, there is little doubt that spontaneous breaking of the chiral symmetry takes place and that, at least in the limit of small quark masses, the effective chiral theory known as Chiral Perturbation Theory $(\chi \mathrm{PT})$ is a valid description of $\mathrm{QCD}[1,2]$ : still, in the former there remain unknown constants ("low-energy constants", or LECs) that only the experiment sensu lato (i.e. including numerical simulations) can constrain (see, e.g., [3] for a review of the relation between $\chi \mathrm{PT}$ and Lattice QCD results).

* Presented at "Excited QCD 2014", Bjelašnica Mountain, Sarajevo, Bosnia and Herzegovina, February 2-8, 2014. 
In this work, data from large-volume two-flavour $\left(N_{\mathrm{f}}=2\right)$ Lattice QCD simulations are used to test $\chi \mathrm{PT}$ and determine some of its LECs. Along the way, different truncations and variations of the predictions from $\chi \mathrm{PT}$ are applied and their practical range of validity is examined. The lattice formulation also introduces possible sources of uncertainty (e.g. the system is necessarily finite and discrete), but this seems to be under control in this investigation.

\subsection{Chiral Perturbation Theory}

$\chi \mathrm{PT}$ is an effective field theory for the low-energy regime of QCD. Its fundamental fields are, in the standard formulation, the pion fields $U$; the theory exhibits spontaneous chiral symmetry breaking by construction, signalled by the fact that the leading-order LEC $\Sigma$, corresponding to the chiral condensate, is nonzero. Together with $F$, the pion decay constant in the chiral limit, those are the two leading-order LECs of the two-flavour $\chi \mathrm{PT}$ (the so-called $\mathrm{SU}(2) \chi \mathrm{PT})$. The theory is order-by-order renormalisable, implying that there are infinitely many LECs: for instance, at next-to-leading order there are seven such energy scales $\Lambda_{n}$, usually expressed with reference to the scale of the physical pion mass: $\bar{\ell}_{n}=\log \Lambda_{n}^{2} / m_{\pi, \text { phys }}^{2}$, for $n=1, \ldots 7$. A distinctive feature of $\chi \mathrm{PT}$ is that its predictions, usually expressed as expansions in small quark masses, small pion masses or analogous, present logarithmic terms (chiral logarithms).

Such is the case for the two formulae that are needed throughout this work (here we stick to the conventions in Section 5.1 of [3]): the quark-mass dependence of the pion mass $m_{\pi}$ and its decay constant $f_{\pi}$, respectively ${ }^{1}$. After adopting an independent variable that is as closely related to lattice measurements as possible, $y=m_{\pi}^{2} /\left(4 \pi f_{\pi}\right)^{2}$ (with the physical point at $\left.y_{\pi} \simeq 0.013\right)$, those two relations, to NNLO, can be written in the form ( $\xi$-expansion)

$$
\begin{aligned}
M_{\pi}^{2}(\beta, y)= & B_{\beta}^{2} \times\left\{1-\frac{1}{2}\left[\bar{\ell}_{3}+2 y_{\pi} \bar{\ell}_{4}+\log y_{\pi}\right] y+\frac{1}{2} y \log y\right. \\
& +\left[c_{M}+\bar{\ell}_{4}+\frac{1}{4}\left(\bar{\ell}_{3}+\log y_{\pi}\right)^{2}+\frac{q_{M}^{2}}{90}\right] y^{2} \\
& \left.-\frac{1}{2}\left[\left(\bar{\ell}_{3}+\log y_{\pi}\right)+\frac{q_{M}}{6}\right] y^{2} \log y+\frac{7}{8} y^{2}(\log y)^{2}\right\} ;
\end{aligned}
$$

\footnotetext{
${ }^{1}$ Throughout this work, lowercase symbols $m$ and $f$ refer to masses and decay constants in physical units, while $M=a m$ and $F=a f$ denote quantities in lattice units, i.e. in appropriate powers of the lattice spacing.
} 


$$
\begin{aligned}
F(\beta, y)= & F_{\beta} \times\left\{1+\left[\bar{\ell}_{4}+\log y_{\pi}+2 y_{\pi} \bar{\ell}_{4}\right] y-1 y \log y\right. \\
& +\left[-c_{F}-2 \bar{\ell}_{4}+\left(\bar{\ell}_{4}+\log y_{\pi}\right)^{2}+\frac{q_{F}^{2}}{36}\right] y^{2} \\
& \left.-\left[2\left(\bar{\ell}_{4}+\log y_{\pi}\right)+\frac{q_{F}}{6}\right] y^{2} \log y+\frac{5}{4} y^{2}(\log y)^{2}\right\} \\
q_{M}= & 60 \bar{\ell}_{12}-33 \bar{\ell}_{3}-12 \bar{\ell}_{4}+52+15 \log y_{\pi} \\
q_{F}= & 18 \bar{\ell}_{4}-15 \bar{\ell}_{12}+3 \log y_{\pi}-\frac{29}{2} .
\end{aligned}
$$

Here, the overall amplitudes are meant in lattice units, hence they will have different values at each of values of the inverse bare coupling $\beta$ for which we have lattice determinations (each $\beta$ is in a one-to-one relation to a lattice spacing). Two LECs appear here at NLO, namely $\bar{\ell}_{3}$ and $\bar{\ell}_{4}$; to NNLO, moreover, three more enter, which are $c_{F}, c_{M}$ and the one associated, similarly as for the other $\bar{\ell}_{m}$, to the combination $\Lambda_{12}^{2}=\left(7 \Lambda_{1}^{2}+8 \Lambda_{2}^{2}\right) / 15$, [4].

\section{Lattice computations}

The masses and decay constants were calculated on configurations generated, within the CLS initiative [6], using the $\mathcal{O}(a)$-improved two-flavour Wilson discretisation of the QCD action. Three lattice spacings $a \simeq 0.075,0.065$, $0.048 \mathrm{fm}$ were simulated in order to get a handle on the continuum limit, corresponding to $\beta=5.2,5.3,5.5$. All systems satisfy the condition $m_{\pi} L \geq 4$ (with $L$ the spatial extent of the discretised system), generally thought to ensure safety from finite-size deviations. The fifteen available ensembles span a range of pion masses down to about $192 \mathrm{MeV}$, along which $\chi \mathrm{PT}$ formulae are fitted.

The pseudoscalar-sector observables $M_{\pi}, F_{\pi}$, as well as the quark mass $M_{q}$, have been extracted by measurements of two-point functions of quark bilinears, which, in turn, are measured through stochastic sampling, as detailed in [5]. The (bare) quantities are then known with typically less-than-percent accuracy (there are renormalisation factors entering afterwards, which somewhat increase the uncertainties at play. $Z_{A}$ alone, in particular, is responsible for about $40 \%$ of the error on the final decay constants). Throughout the analysis, errors are propagated carefully and the methods developed in [7] are applied in order not to underestimate hidden "slow modes" in the Monte Carlo chains. 
For the translation into physical quantities, scale-setting is a necessary last ingredient. Usually, this is done by measuring a high-precision quantity on the lattice, say the kaon decay constant $F_{K}(\beta)$, and then using the experimental value $f_{K}=155 \mathrm{MeV}$ to get $a(\beta)=F_{K} / f_{K}$ in fermi (note that here $\left|V_{u s}\right|$ also enters). This was the procedure followed in this analysis: the previous experience shows that the kaon decay constant provides a rather robust scale and allows for less-than-percent precisions on $a(\beta)$. We refer to [5] for the technical details on how valence $s$-quarks are added to a theory with only the $(u, d)$ pair as dynamical quark content (partial quenching).

\section{Chiral fits to $M_{\pi}, F_{\pi}$}

In practice, instead of Eq. (1), the equivalent combination $\rho \equiv M_{\pi}^{2} /\left(2 M_{q}\right)$ is built and fitted to an analogous chiral formula with just a different amplitude in front: in the continuum, this amplitude would be just $a^{3} \Sigma / F^{2}$, with $\Sigma$ representing the chiral condensate in physical units. Moreover, the data are not on the continuum, hence a further term in the overall amplitude is added modelling the $a^{2}$ discretisation effects. Finally, for the sake of convenience, we rewrite this amplitude so as to obtain, directly as a fit parameter, the adimensional ratio of $\Sigma$ to the physical pion decay constant cube, $S_{0}=\Sigma / f_{\pi, \text { phys }}^{3}$. The NNLO fit function for $\rho$, then, is analogous to Eq. (1), but in place of the overall $B_{\beta}^{2}$, we have the prefactor

$$
\begin{aligned}
\sigma_{\beta}= & \left(S_{0}+F_{\beta}^{2} S_{1}\right) F_{\beta}\left\{1+y_{\pi} \bar{\ell}_{4}+y_{\pi}^{2}\left(\bar{\ell}_{4}^{2}+\frac{q_{F}^{2}}{36}-c_{F}\right)\right. \\
& \left.+y_{\pi}^{2} \log y_{\pi}\left(-\frac{1}{6} q_{F}\right)+\frac{1}{4} y_{\pi}^{2}\left(\log y_{\pi}\right)^{2}\right\}^{3},
\end{aligned}
$$

the term in curly brackets expressing $f_{\pi}\left(y_{\pi}\right) / f$ as per Eq. (2) (or variations thereof when needed). The two datasets, $F_{\pi}$ and $M_{\pi}$, are fitted simultaneously, and for $F_{\beta}$ we still have three different values, one per each $\beta$.

Three families of functional forms are attempted: (a) the NNLO formulae shown so far; (b) their NLO truncation, i.e. limited to terms of the order of $y$ and $y \log y$; and (c) the so-called "junction" formulae. The latter come from the empirical observation that pion observables, in the available range, seem to just lie on a straight line: on the other hand, we expect $\chi \mathrm{PT}$, close enough to the origin, to take over; hence, we consider a linear function of $y$ beyond some "junction point" $y>y_{\text {jct }}$, and the NLO formulae on the left, with continuity, up to the first derivative, in $y_{\text {jct }}$. The total number of fit parameters is 10 for (a) and 7 for (b) and (c). Also different pionmass fit ranges are explored, namely $m_{\pi} \leq 650,500,390$ and $345 \mathrm{MeV}-$ 
corresponding to using 15, 12, 8 and 7 ensembles, respectively (and each ensemble provides two data points). Representative fits to the datapoints are shown in Fig. 1.
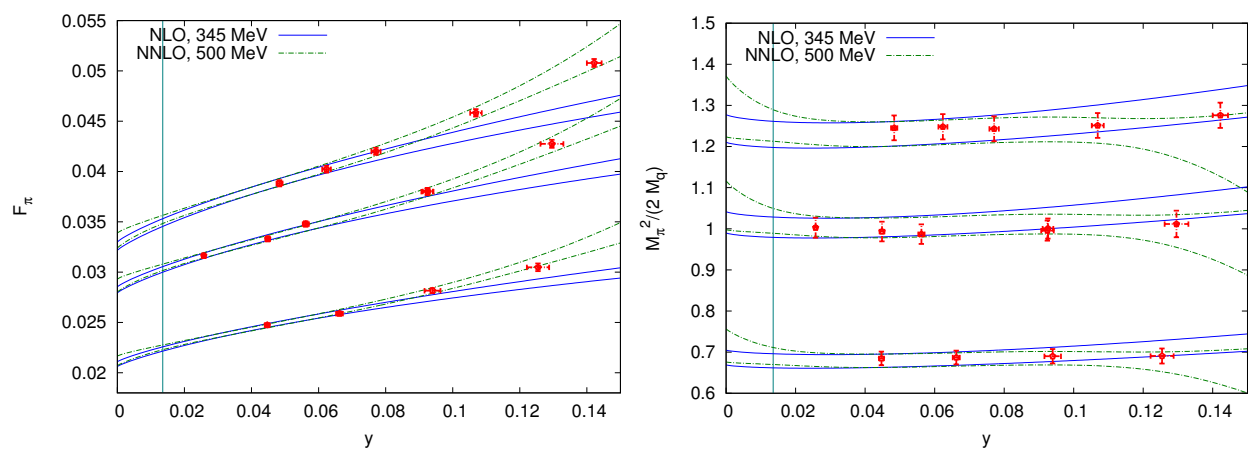

Fig. 1. Chiral behaviour of $F_{\pi}$ (left) and $M_{\pi}^{2} /\left(2 M_{q}\right)$ (right) as a function of $y$. Each dataset corresponds to a value of $\beta$ (the topmost line being for the largest lattice spacing). A one-sigma band is shown for the NLO fit with mass-cutoff $345 \mathrm{MeV}$ and for the NNLO cut at $500 \mathrm{MeV}$. The vertical line marks $y_{\pi}$.

We focus on the results for: $F_{\beta}$ (and the continuum-limit physical value $f_{\pi}$ that stems from it), $\Sigma^{1 / 3}$, the higher-order LECs $\bar{\ell}_{3}, \bar{\ell}_{4}, \bar{\ell}_{12}$; the chiral condensate, as well as the quark mass $m_{q}$, are here always understood to be expressed in the $\overline{\mathrm{MS}}$ scheme at scale $\mu=2 \mathrm{GeV}$. All fit variants performed will enter an assessment of the systematic uncertainties involved, generally of higher magnitude than the similar analysis performed in the kaon sector. Inspection of "stability plots" (i.e. pion-mass-cutoff dependence of the fit parameters, see Fig. 2) suggests that, as far as $F_{\pi}$ is concerned, NLO with low mass-cut coincides with NNLO, hence we trust the former, with the lowest
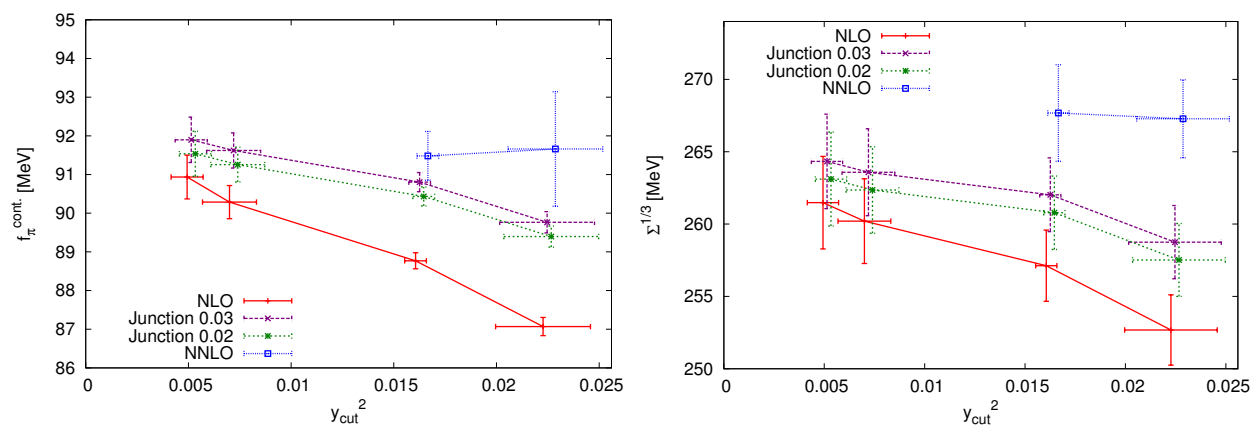

Fig. 2. "Stability plots" for selected fit results: $y_{\text {cut }}$ (i.e. pion-mass-cutoff) dependence of $F_{\beta=5.3}$ (left) and of $\Sigma^{1 / 3}$ (right) for the various fit procedures. The horizontal axis is $y_{\text {cut }}^{2}$, which should roughly make the points lie on a straight line. 
mass-cut, and take its continuum limit (upon insertion of the $F_{K}$-based scalesetting), which yields $f_{\pi}=91.7(1.5)\left({ }_{-0.7}^{+0.2}\right) \mathrm{MeV}^{2}$. Also the chiral condensate depends weakly on the fit procedure and, again, we use the NLO fit and quote $\Sigma^{1 / 3}=261.5(3.2)(5.8) \mathrm{MeV}$, a value compatible with the world-average recently reported in [3]. A computation of $\Sigma$, based on condensation of Dirac modes near zero, will be reported in [8].

As for the higher-order LECs, their precise determination is notoriously very demanding (see, e.g., [4] for a recent similar analysis), in particular due to changes as one adjusts slightly the fit strategy: we therefore just remark that NNLO fits are unable to give estimates which are not compatible with zero within their errors, and report the best results - again from NLO fits at minimum pion-cut - for: $\bar{\ell}_{3}=1.3(0.8)$ and $\bar{\ell}_{4}=4.9(0.7)$. We refer to a forthcoming publication for a more detailed, conclusive analysis of these $N_{\mathrm{f}}=2$ data, and, in particular, an assessment of the systematic uncertainties on the NLO constants.

\section{Conclusions}

We find that the two-flavour Lattice QCD measurements in the pion sector using $\chi \mathrm{PT}$-inspired formulae are much less straightforward to analyse than the analogous ones in the kaon sector (with the $s$ a valence quark). A general conclusion is that the range of validity of NLO $\chi \mathrm{PT}$ (if not of $\chi \mathrm{PT}$ tout court) is shorter, which prompts the use and comparison of several possible fit functions. Even so, as soon as one turns their attention to NLO low-energy constants the uncertainties (especially the systematic ones) are rather large. In order to provide a thorough characterisation of two-flavour QCD in terms of its LECs, then, it would be desirable to include more ensembles with even smaller pion masses; however, production of $N_{\mathrm{f}}=2$ CLS ensembles has been discontinued in favour of $N_{\mathrm{f}}=2+1$ systems, where presumably similar issues will be seen.

The Author gratefully acknowledges the Conference Organisers for their patience and for providing such an enjoyable and informal atmosphere, as was the case in previous editions of Excited QCD as well.

\section{REFERENCES}

[1] S. Weinberg, Physica A 96, 327 (1979).

[2] J. Gasser, H. Leutwyler, Ann. Phys. 158, 142 (1984).

[3] S. Aoki et al., arXiv:1310.8555 [hep-lat].

${ }^{2}$ The first error is statistical, the second is systematic. Note that some conventions keep an additional factor $\sqrt{2}$ into $f_{\pi}$. 
[4] S. Dürr et al., arXiv:1310.3626 [hep-lat].

[5] P. Fritzsch et al., Nucl. Phys. B865, 397 (2012) [arXiv: 1205.5380 [hep-lat]].

[6] https://wiki-zeuthen.desy.de/CLS/CLS

[7] S. Schaefer et al. [ALPHA Collaboration], Nucl. Phys. B845, 93 (2011) [arXiv:1009.5228 [hep-lat]].

[8] G.P. Engel, L. Giusti, S. Lottini, R. Sommer, arXiv:1406.4987 [hep-ph]. 